\title{
原著論文
}

\section{Postnatal changes in the repetitive firing properties of rat jaw-closing motoneurons}

\author{
Tomio Inoue $^{1)}$, Akiko Yamaoka ${ }^{2)}$, Shoji Hironaka ${ }^{3)}$, Shiro Nakamura ${ }^{1)}$ \\ Kiyomi Nakayama $^{1)}$, Ayako Mochizuki ${ }^{1)}$, Yoshiharu Mukai ${ }^{3)}$, Kazuo Itabashi $^{2)}$ \\ ${ }^{1)}$ Departments of Oral Physiology, School of Dentistry, Showa University \\ ${ }^{2)}$ Department of Pediatrics, School of Medicine, Showa University \\ ${ }^{3)}$ Departments of Hygiene and Oral Health, School of Dentistry, Showa University
}

[Received: August 15, 2012]

Key words: development, trigeminal, motoneuron, jaw-closing, afterdepolarization

\begin{abstract}
Feeding behavior drastically changes from suckling to chewing during the postnatal period. Thus, it is possible that the firing properties of jaw-closing motoneurons (JCMNs), one of the final common pathways in the oro-motor system, are also altered during the postnatal period. We examined the repetitive firing properties of JCMNs in developing rats from an early postnatal stage to a post-weaning stage using intracellular recording techniques. Firing frequency adaptation was observed within the first four spikes in a spike train. The neurons were classified into two groups according to the firing patterns subsequent to the first four spikes: neurons with a constant or slightly decrementing firing pattern (type I) and neurons with an incrementing firing pattern (type II). The percentage of type I neurons increased with age. In more than 50\% of the neurons in each age group except for the group at 10-15 days of age, an extra spike was produced from the post-spike afterdepolarization (ADP) of the 1st spike in a spike train, leading to a high firing rate for the first inter-spike interval (1st ISI). The incidence of an extra spike also increased with age. The firing rate for the steady-state was high in JCMNs, even at 4-9 days of age, and approximately 50\% of the neurons at this age fired at higher than $50 \mathrm{~Hz}$. These findings demonstrate that JCMNs can fire at a high rate, even in the early postnatal period, and the firing rate for the 1st ISI further increases with age. These changes may contribute to the transition from suckling to chewing.
\end{abstract}

連絡先： $=142-8555$ 東京都品川区旗の台 $1-5-8$

TEL: 03-3784-8159 FAX: 03-3784-8161 


\section{Introduction}

Feeding behavior in mammals drastically changes from suckling to chewing during the early postnatal period. The activity of the jaw-closing muscle should be low during the suckling phase, whereas the activity increases when hard or tough food is chewed after early postnatal development ${ }^{1-4)}$. Thus, motor commands to jaw-closing muscles change with development of the orofacial musculoskeletal system during the early postnatal period. It is possible that properties of the jaw-closing motoneurons (JCMNs), one of the final common pathways in the oro-motor system, are also altered with changes in the motor commands. We previously reported that the action potential half-duration, and the amplitude and half-duration of the medium-duration afterhyperpolarization (mAHP) decreased during postnatal development; in contrast, the incidence of post-spike afterdepolarization (ADP) progressively increased with the age of the $\mathrm{JCMN}^{5}$. Furthermore, we reported that glycinergic and/or GABAergic inputs from the supratrigeminal nucleus and the reticular formation dorsal to the facial nucleus change from excitatory to inhibitory during postnatal development ${ }^{6,7)}$. However, the postnatal changes in firing properties of JCMNs are still unclear.

In this study, we used intracellular recordings to investigate the firing properties of JCMNs that innervate the jawclosing muscle in brainstem slice preparations containing the trigeminal motor nucleus of developing rats from an early postnatal stage to the post-weaning stage. We demonstrate that JCMNs can be classified into two groups according to firing patterns after the first four spikes, including neurons with a constant or slightly decrementing firing pattern (type I) and neurons with an incrementing firing pattern (type II). In addition, the percentage of type I neurons and the incidence of an extra spike increase with age. These changes may contribute to the transition from suckling to chewing.

\section{Materials and Methods}

All experiments were approved by the International Animal Research Committee of Showa University in accordance with Japanese Government Law \#105 concerning the care and use of laboratory animals. All efforts were made to minimize the number of animals that were used.

\section{Slice preparation}

Experiments were performed with brainstem slices obtained from P3-27 Wistar rats $(n=52)$. The day of birth was defined as P0. Neonatal (P3-P15) and juvenile (P16-P27) rats were deeply anesthetized by ether or combined injections of ketamine $\mathrm{HCl}$ (150 mg/kg i.m.) and chlorpromazine $\mathrm{HCl}$ (12.5 $\mathrm{mg} / \mathrm{kg}$ i.m.), respectively. Following decapitation, the brain was removed rapidly and placed in cold oxygenated sucrosebased modified artificial cerebrospinal fluid (ACSF) because it has been shown that use of this ACSF during the slicing procedure is essential to maintain viable motoneurons in adult rats $^{8)}$. The modified ACSF contained (in $\mathrm{mM}$ ) 260 sucrose, $3 \mathrm{KCl}, 2 \mathrm{CaCl}_{2}, 2 \mathrm{MgCl}_{2}, 1.25 \mathrm{NaH}_{2} \mathrm{PO}_{4}, 26 \mathrm{NaHCO}_{3}$ and 10 glucose. The slices were incubated at $34^{\circ} \mathrm{C}$ in a holding chamber containing modified ACSF for 15 min, transferred to a holding chamber containing normal ACSF (N-ACSF) at $34^{\circ} \mathrm{C}$ for $30 \mathrm{~min}$ and were maintained at room temperature. $\mathrm{N}$-ACSF contained (in $\mathrm{mM}$ ) $130 \mathrm{NaCl}, 3 \mathrm{KCl}, 2 \mathrm{CaCl}_{2}, 2$ $\mathrm{MgCl}_{2}, 1.25 \mathrm{NaH}_{2} \mathrm{PO}_{4}, 26 \mathrm{NaHCO}_{3}$ and 10 glucose.

\section{Recordings}

To identify the recorded cell as a JCMN, we used a fluorescence labeling technique ${ }^{5)}$. One to two days before the slices were prepared, animals were anesthetized with ether or combined injection of ketamine $\mathrm{HCl}$ and chlorpromazine $\mathrm{HCl}$, and $2-5 \mu \mathrm{l}$ of $10 \% 3,000$ or $10,000 \mathrm{MW}$ dextrantetramethylrhodamine-lysine (DRL, Molecular Probes, Eugene, OR, USA) dissolved in distilled water was injected bilaterally in the masseter muscles with a microsyringe (1010RN, Kloehn, Las Vegas, NV, USA).

After a 2-10 $\mathrm{h}$ incubation in the holding chamber, the slices were transferred to an interface-type chamber. The recording chamber was continuously perfused with N-ACSF at a rate of $1-1.5 \mathrm{ml} / \mathrm{min}$, and an atmosphere consisting of humidified $95 \% \mathrm{O}_{2}-5 \% \mathrm{CO}_{2}$ flowed over the slice. All experiments were performed at $32 \pm 1^{\circ} \mathrm{C}$. Intracellular recordings were obtained with glass microelectrodes (1.2 mm O.D., 20-40 M.) (Sutter Instruments, Novato, CA, USA) filled with $1 \%$ biocytin in $1 \mathrm{M} \mathrm{KCl}$ and $0.05 \mathrm{M}$ Tris buffer $(\mathrm{pH}$ 7.6). The trigeminal motor nucleus was visible in the slice through the stereomicroscope (MZ FL III, Leica Microsystems GmbH, Wetzlar, Germany). The neurons retrogradely labeled by DRL were visualized with an epifluorescence unit attached to the microscope and localized in the dorsolateral portion of the trigeminal motor nucleus. Next, the electrode was 
Table 1 Membrane and firing properties of jaw-closing motoneurons

\begin{tabular}{|c|c|c|c|c|c|c|}
\hline & \multicolumn{6}{|c|}{ Age, days } \\
\hline & $4-9$ & $10-15$ & $16-21$ & $22-27$ & $4-15$ & $16-27$ \\
\hline $\begin{array}{l}\text { Resting } \\
\text { membrane potential }(\mathrm{mV})\end{array}$ & $\begin{array}{l}-68.3 \pm 1.4 \\
(17)\end{array}$ & $\begin{array}{l}-70.0 \pm 1.4 \\
(24)\end{array}$ & $\begin{array}{l}-62.7 \pm 1.8 \\
\quad(7)\end{array}$ & $-68.8 \pm 1.50$ & $\begin{array}{l}-69.3 \pm 1.0 \\
(41)\end{array}$ & $\begin{array}{l}-65.2 \pm 1.8 \\
\quad(11)\end{array}$ \\
\hline Spike amplitude $(\mathrm{mV})$ & $\begin{array}{l}87.4 \pm 1.4 \\
\quad(17)\end{array}$ & $\begin{array}{l}83.9 \pm 0.9 \\
\quad(24)\end{array}$ & $\begin{array}{l}83.6 \pm 1.7 \\
\quad(7)\end{array}$ & $\begin{array}{l}87.2 \pm 1.50 \\
\quad(4)\end{array}$ & $\begin{array}{l}85.3 \pm 0.8 \\
\quad(41)\end{array}$ & $\begin{array}{l}83.5 \pm 1.2 \\
\quad(11)\end{array}$ \\
\hline \multicolumn{7}{|l|}{ Number of neurons } \\
\hline type I & $9(52.9 \%)$ & $10(41.7 \%)$ & $6(85.7 \%)$ & $3(75.0 \%)$ & $19(46.3 \%)$ & $9(81.8 \%)$ \\
\hline type II & $6(35.3 \%)$ & $13(54.2 \%)$ & $1(14.3 \%)$ & $1(25.0 \%)$ & $19(46.3 \%)$ & $2(18.2 \%)$ \\
\hline others & $2(11.8 \%)$ & $1(4.2 \%)$ & $0(0.0 \%)$ & $0(0.0 \%)$ & $3(7.3 \%)$ & $0(0.0 \%)$ \\
\hline 1st ISI f-I slope (Hz/nA) & $\begin{array}{l}36.8 \pm 6.2 \\
\quad(17)\end{array}$ & $\begin{array}{l}38.6 \pm 3.2 \\
\quad(24)\end{array}$ & $\begin{array}{c}35.4 \pm 3.8 \\
(7)\end{array}$ & $\begin{array}{l}44.9 \pm 7.3 \\
\quad(4)\end{array}$ & $\begin{array}{l}38.5 \pm 3.1 \\
\quad(41)\end{array}$ & $\begin{array}{l}38.9 \pm 3.7 \\
\quad(11)\end{array}$ \\
\hline Steady-state f-I slope $(\mathrm{Hz} / \mathrm{nA})$ & $\begin{array}{l}22.3 \pm 2.0 \\
\quad(17)\end{array}$ & $\begin{array}{l}22.7 \pm 2.0 \\
\quad(24)\end{array}$ & $\begin{array}{l}19.1 \pm 2.5 \\
\quad(7)\end{array}$ & $\begin{array}{l}30.2 \pm 3.7 \\
\quad(4)\end{array}$ & $\begin{array}{l}24.2 \pm 1.3 \\
\quad(41)\end{array}$ & $\begin{array}{l}23.1 \pm 2.6 \\
\quad(11)\end{array}$ \\
\hline Incidence of extra spike & $\begin{array}{c}9 / 13 \\
(69.2 \%)\end{array}$ & $\begin{array}{c}7 / 16 \\
(43.7 \%)\end{array}$ & $5 / 6(83.3 \%)$ & $4 / 4(100 \%)$ & $\begin{array}{c}16 / 29 \\
(55.2 \%)\end{array}$ & $9 / 10(90.0 \%)$ \\
\hline Incidence of secondary range & $\begin{array}{c}6 / 13 \\
(46.2 \%) \\
\end{array}$ & $\begin{array}{c}4 / 16 \\
(25.0 \%) \\
\end{array}$ & $4 / 6(66.7 \%)$ & $2 / 4(50 \%)$ & $\begin{array}{c}10 / 29 \\
(34.5 \%)\end{array}$ & $6 / 10(60.0 \%)$ \\
\hline
\end{tabular}

inserted into the cluster of DRL-labeled neurons in the slice. Following recording, we re-confirmed whether the tip of the electrode was located within the cluster of labeled neurons. It has been shown that motoneurons innervating the jaw-closing muscles, such as the masseter or temporalis muscles, are localized in the dorsolateral portion of the trigeminal motor nucleus of the rat, and neurons innervating the jaw-opening muscles, such as the anterior digastric or mylohyoid muscles, are located in the ventromedial portion ${ }^{9,10)}$. Furthermore, there are minimal interneurons in the trigeminal motor nucleus ${ }^{11)}$. Thus, we regarded the neurons located within a cluster of DRL-labeled neurons as JCMNs. In some neurons, we further confirmed if the recorded neurons were localized in a cluster of DRL-labeled neurons using histological observations (cf. Yamaoka et al. $\left.{ }^{5}\right)$. Membrane potentials were recorded using an Axoclamp 2B amplifier (Molecular Devices, Sunnyvale, CA, USA) in bridge mode (see Inoue et al. ${ }^{12)}$ ). The membrane potential was filtered at $30 \mathrm{kHz}$ with built-in single-pole lowpass filters, digitized at $40 \mathrm{kHz}$ and stored on a computer hard disk using software (Clampex, Molecular Devices) through an A-D converter. Data were analyzed with Clampfit (Molecular Devices) and Excel (Microsoft Japan, Tokyo) software. Fiftytwo JCMNs were used to analyze the electrophysiological properties, including 4 postnatal age groups: 4-9 days $(n=$
17), 10-15 days $(n=24), 16-21$ days $(n=7)$ and 22-27 days $(n=4)$. To examine the properties of repetitive firing, trains of spikes were elicited in JCMNs by intracellular injections of long (1 s) depolarizing current pulses. The instantaneous firing frequency was defined as the reciprocal of the interspike interval (ISI), and the steady-state firing frequency was calculated as the average of the firing rate over the last half (500 ms) of the injected current pulse.

Data are presented as the mean \pm SE. Comparisons of data between groups were completed using Student's $t$-test, Fisher's exact test or the $\chi^{2}$ test. Probabilities $<0.05$ were considered significant.

\section{Results}

\section{Repetitive firing patterns}

The present study was based on recordings from 52 JCMNs that had stable resting potentials more negative than $-55 \mathrm{mV}$ ($68.4 \pm 0.9 \mathrm{mV})$ and displayed spikes larger than $70 \mathrm{mV}(85.0$ $\pm 0.7 \mathrm{mV}$ ). There were no significant differences in the resting potentials and spike amplitudes among the four age groups (Table 1).

Trains of spikes could be elicited in developing JCMNs $(n=52)$ by $1 \mathrm{~s}$ depolarizing current pulses (Fig. $1 A, D)$. 
A

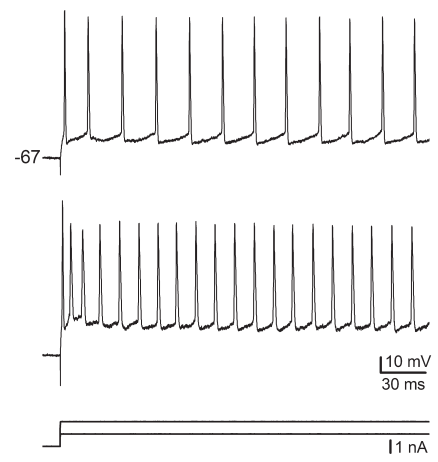

D

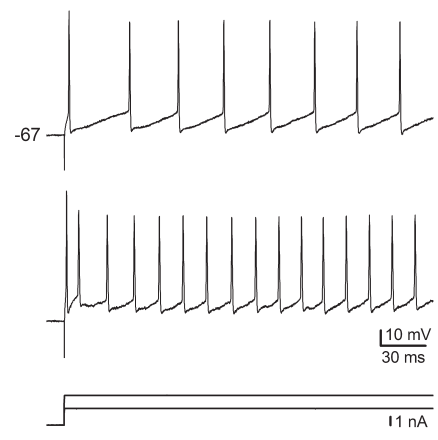

B

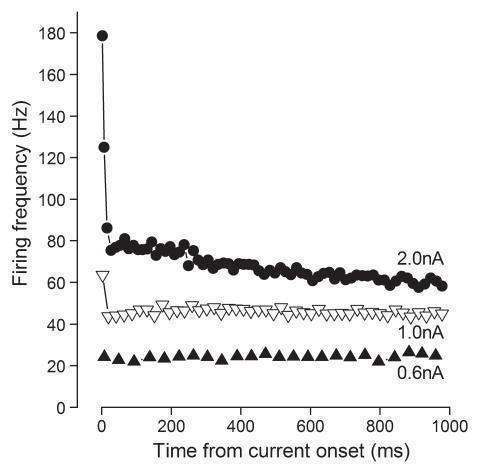

E

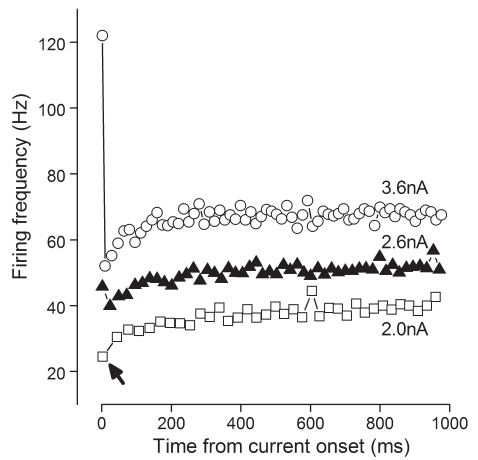

C

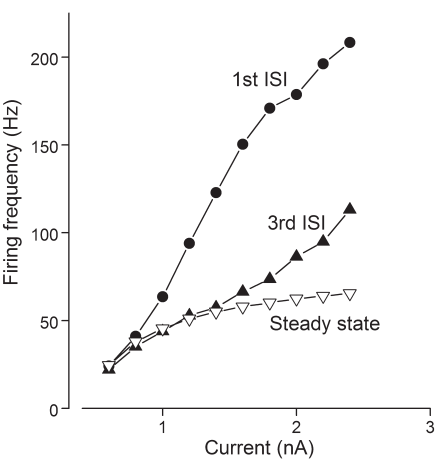

$\mathrm{F}$

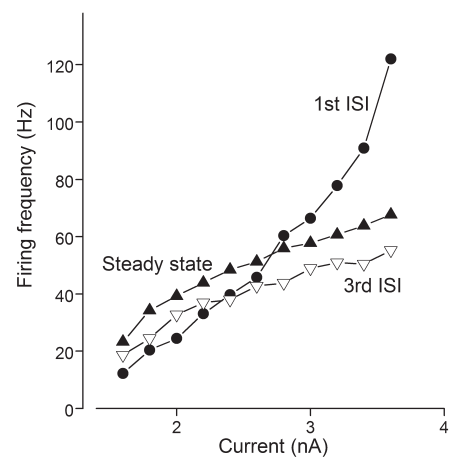

Fig. 1 Repetitive firing characteristics of type I and II JCMNs.

A: Repetitive firing of an 8-day-old type I neuron induced by 1-s constant current pulses at $1.0 \mathrm{nA}$ (upper) and $2.0 \mathrm{nA}$ (lower). $B$ : Frequency-time plot $(f-t$ plot) for the neuron in $A$ for 3 different current intensities. Note that there is a prominent early adaptation observed at current intensities of 1.0 and $2.0 \mathrm{nA}$.

$C$ : Frequency-current $(f-I)$ relationship for 1st ISI, 3rd ISI and steady-state firing for the neuron in $A$.

$D$ : Repetitive firing of a 6-day-old type II neuron at $2.0 \mathrm{nA}$ (upper) and $3.6 \mathrm{nA}$ (lower) current intensities.

$E$ : Frequency-time plot ( $f$ - $t$ plot) for the neuron in $D$. Note that the firing rate for the 1st ISI (arrow) was lowest in the spike train at a $2.0 \mathrm{nA}$ current intensity; however, prominent early adaptation appeared at $3.6 \mathrm{nA}$.

$F: f-I$ relationship for the neuron in $D$. Note that $f-I$ plots for the 1 st ISI and steady-state intersect at approximately $2.7 \mathrm{nA}$.

The instantaneous firing rate was plotted against the time of occurrence of each spike $(f-t$ plot) to analyze the firing properties (Fig. 1B, E). At moderate to high current intensities, the firing rates for the 1st ISIs were highest in the spike trains of all neurons tested, and they rapidly decreased by the fourth ISI at the latest (early adaptation). The subsequent firing patterns were different among the neurons and were classified into 2 groups. Type I neurons showed relatively constant or slowly decreasing firing during the current pulse (Fig. $1 B, n$ $=28,55 \%$ of the analyzed cells), and type II neurons showed a rapid acceleration of firing rate for 100-200 ms and a subsequent constant or slowly increasing firing rate (Fig. $1 E$, $n=21,41 \%)$. In $25 \%(7 / 28)$ of type I neurons, the firing rate continued to decrease after early adaptation at high current intensities (Fig. 1B $2.0 n A$ ). In 47\% (10/21) of type II neurons, the firing rate for the 1st ISI was lowest in the spike train at low current intensities (Fig. $1 D$ upper and $1 E$ arrow). Even in these 10 type II neurons, the firing rate for the 1 st ISI became highest among the rate for the subsequent ISIs during higher current pulses (Fig. $1 D$ lower and $E$ 3.6 $n A$ ). The increase in the firing rate for the 1st ISI corresponded to the steeper slope of the relationships between the firing frequency and injected 
Table 2 Relationships between the repetitive firing properties and incidences of extra spike and secondary range

\begin{tabular}{lcccc}
\hline & type I & type II & others & Total \\
\hline Incidence of extra spike & $12 / 23(52.1 \%)$ & $11 / 14(78.5 \%)$ & $2 / 2(100 \%)$ & $25 / 39(64 \%)$ \\
Incidence of secondary range & $11 / 23(47.8 \%)$ & $5 / 14(35.7 \%)$ & $0 / 2(0 \%)$ & $16 / 39(41 \%)$ \\
\hline
\end{tabular}

current ( $f-I$ relationships) for the 1 st ISI, compared with the slopes for the 3rd ISI and steady-state (Fig. $1 F$ ). The ratio of the 1 st ISI slope to the steady-state slope was $1.96 \pm 0.14(n=$ 10). In the remaining 3 neurons, which were neither type I nor II, two neurons had a type II firing pattern during a smaller injected current and a type I firing pattern during a larger injected current. One neuron had a complex firing pattern.

At the age of 4-9 and 10-15 days the numbers of type I and type II neurons were similar; however, the majority of JCMNs ( $\geq 75 \%$ ) were type I neurons at 16-21 and 22-27 days (Table 1). When the data were pooled from 4- to 15-day-old animals and from 16- to 27-day old animals, the percentage of type I neurons in 16- to 27-day old animals (81.8\%) was significantly greater than the percentage of type I neurons $(46.3 \%)$ in younger animals (Fisher's exact test, $P<0.05$ ). Neither the $f$-I slope for the 1st ISI nor did steady-state differ among the age groups (Table 1).

JCMNs could also fire at a high rate in the steady-state. Although we did not inject currents into all neurons large enough to determine the maximum steady-state firing rates, the steady-state firing rate exceeded $50 \mathrm{~Hz}$ at current intensities of 1.5-4.0 nA in 54\% (28/52) of the neurons tested. Even at 4-9 days, approximately 50\% (8/17) of the neurons fired at higher than $50 \mathrm{~Hz}$, and the firing rate exceeded $67 \mathrm{~Hz}$ in 3 of the 8 neurons.

\section{Production of an extra spike}

When we injected large currents up to $4.0 \mathrm{nA}$ into 39 JCMNs, an extra spike was generated from the ADP of the 1st spike in $64 \%(25 / 39)$ of the neurons (Table 1, 2). Figure $2 C c$ shows an example of the extra spike obtained from a 4-dayold JCMN. This neuron showed only a small depolarizing hump following a single action potential elicited by an injection of a short current pulse (Fig. 2A); however, a distinct ADP was induced by a long current pulse at low intensity (Fig. $2 \mathrm{Ca}$ arrow). At higher current intensities, the peak potential of the 1st ADP in the spike train became more depolarized (Fig.
$2 \mathrm{Cb})$ and exceeded the spike threshold, leading to generation of an extra spike from the 1st ADP (Fig. 2Cc arrowhead). In this neuron, the $f$ - $I$ slope for the 1st ISI before occurrence of the extra spike did not differ from the slope in the presence of the extra spike. An extra spike was also obtained from older JCMNs (Fig. 2B, $D$ and $F$ ). When the data were pooled from 4- to 15-day-old animals and from 16- to 27-day old animals, the incidence of an extra spike was significantly higher in 16to 27-day old animals than in 4- to 15-day-old animals (Fisher' s exact test, $P<0.05$ ) (Table 1$)$. Furthermore, when an extra spike was generated, the firing rate for the 1st ISI jumped to significantly higher value in 16-27-day-old-rats than in 415-day-old-rats $(188.5 \pm 18.1 \mathrm{~Hz}, n=9$ vs. $108.8 \pm 8.76 \mathrm{~Hz}$, $n=16 ; P<0.005$; points indicated by 'c' in Fig. $2 E$ and $F$ ). The incidence of the extra spike was not different between type I and type II neurons ( $\chi^{2}$ test, $P>0.10$, Table 2$)$. In the remaining 14 neurons that did not display an extra spike, 6 neurons had a distinct ADP following the action potential elicited by a short current pulse; however, the peak potential of the 1st ADP of these 6 neurons did not exceed the spike threshold, even when firing rate for the 1 st ISI increased to 70 $-120 \mathrm{~Hz}$ (data not shown).

Sixteen neurons $(41 \%)$ of the 39 neurons have bilinear $f-I$ relationships for the 1 st ISI, exhibiting a distinct primary and a steeper secondary range of firing (Fig. $2 F$ ) previously described in adult brainstem motoneurons ${ }^{13-16)}$. In contrast to earlier reports, we found that a steeper secondary range of the 1st ISI was observed in neurons from all age groups (Table 1). The incidence of the secondary range was not significantly different between age groups when the data were pooled from 4- to 15-day-old animals and from 16- to 27-day old animals ( $\chi^{2}$ test, $P>0.10$ ). The occurrence of the secondary range was related to neither type I/type II classification $\left(\chi^{2}\right.$ test, $P>0.25$, Table 2) nor the incidence of the extra spike $\left(\chi^{2}\right.$ test, $\left.P>0.05\right)$. 
A

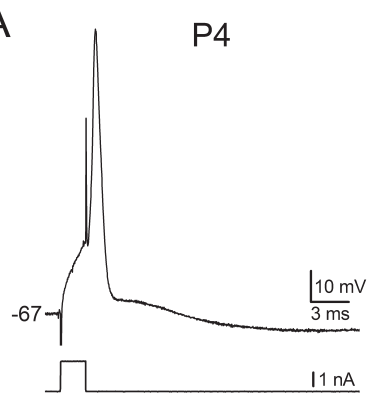

$\mathrm{C}_{\mathrm{a}}$
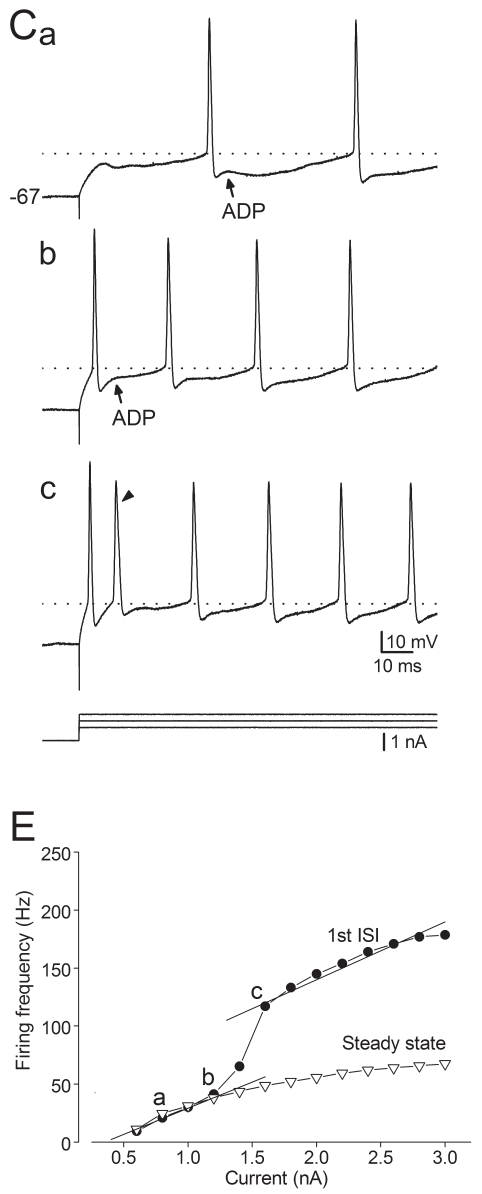

B
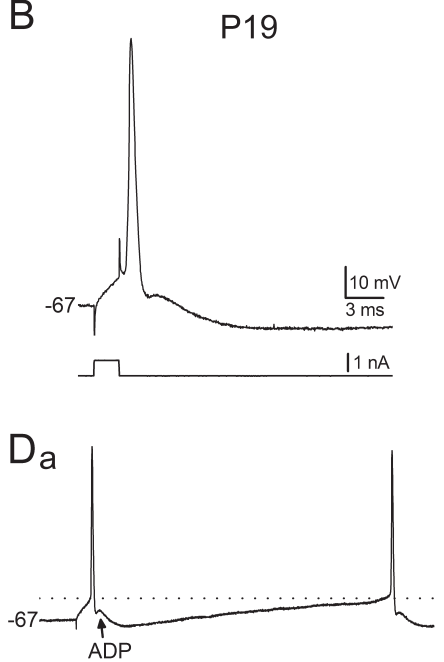

b

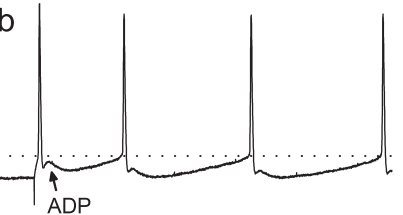

C

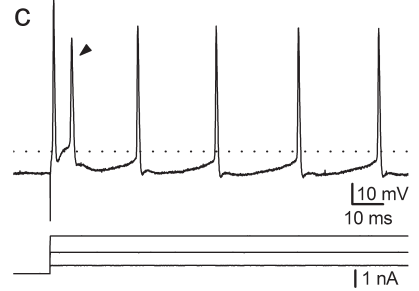

F

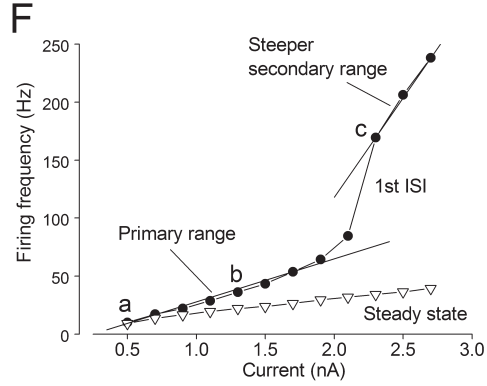

Fig. 2 Generation of an extra spike from the 1st ADP in the spike train.

$A, B$ : Action potentials from 4- $(A)$ and 19-day-old $(B)$ type I neurons. Note that a distinct ADP was not observed in 4-day-old neurons.

$C$ : Repetitive firing was evoked by current injection at 0.7 (a), 1.2 (b) and 1.6 (c) nA for the neuron in $A$. Note that the extra spike generated from the 1st ADP (arrowhead).

$D$ : repetitive firing was evoked by current injection at 0.5 (a), 1.3 (b) and 2.3 (c) nA for the neuron in $B$.

$E: f-I$ relationship for the neuron in $A$. The points on the graph indicated by 'a-c' were obtained from the corresponding traces in $C$.

$F: f-I$ relationship for the neuron in $B$. The points on the graph indicated by 'a-c' were obtained from the corresponding traces in $D$. The dotted lines in $C$ a-c and $D a-c$ indicate the spike threshold of the 1st spike in $C$ a and $D$ a, respectively. 


\section{Discussion}

Repetitive firing patterns of JCMNs were characterized by the higher firing rate for the 1st ISI than the rate for succeeding ISIs (early adaptation), as observed in trigeminal ${ }^{17)}$, genioglossal $^{15)}$, and spinal ${ }^{18)}$ motoneurons. Regarding firing patterns following early adaptation, type II neurons showed an increasing firing pattern and were found in 4-15-day-oldrats. In hypoglossal motoneurons, the majority of the cells in 8-15-day-old rats show an accelerating firing pattern, the $f$ - $I$ slope for the steady-state is steeper than the slope for the 1st ISI, and the firing rate for steady-state is higher than the rate for the 1st ISI, even at large current intensities ${ }^{16)}$. In contrast, when current pulses at high intensities were injected into type II JCMNs, the firing rate for the 1st ISI became highest in the spike train, and the $f-I$ slope for the 1 st ISI was steeper than the slope for the steady-state (Fig. 1F). Furthermore, the number of type II neurons was not different from the number of type I neurons in 10-15-day-old rats. Therefore, the firing properties of type II JCMNs were different from the hypoglossal motoneurons regarding the accelerating firing pattern.

It has been reported that maximal steady-state firing frequency of motoneurons is low in neonatal animals compared with adults ${ }^{15,16,18)}$. The maximal rate is $33 \mathrm{~Hz}$ with a range of $19-44 \mathrm{~Hz}$ in neonatal rat spinal motoneurons ${ }^{18)}$, and the rate exceeds $50 \mathrm{~Hz}$ in only one of 51 neonatal (015-day-old) rat hypoglossal motoneurons ${ }^{16)}$. In contrast to these motoneurons, approximately 50\% JCMNs at 4-9 days fired at higher frequencies than $50 \mathrm{~Hz}$. The amplitude and half duration of the mAHP in JCMNs at 4-9 days are slightly smaller compared with hypoglossal motoneurons ${ }^{5)}$. Thus, a high steady-state firing rate may be due to the smaller mAHP because AHP is an important factor in the control of neuronal discharge ${ }^{19)}$. Furthermore, trigeminal motoneurons have been reported to display persistent sodium currents during the membrane depolarization ${ }^{17}$. These persistent inward currents might have increased the steady-state firing frequency in neonatal JCMNs.

As mentioned above, the maximal firing rate in the steadystate was high in JCMNs from all age groups. Although generation of a large force by the jaw-closing muscles is not necessary during suckling, it is likely that JCMNs were preparing for producing relatively robust force early in postnatal life. In fact, when anything is placed in the mouth, human infants show the bite reflex (a sudden closure of the mandible with activation of the masseter muscle), even at birth $^{20)}$. Extensive occlusal wear of the molar teeth is found in guinea pig fetuses, even ten days prior to birth ${ }^{21)}$. These results suggest that neonates before weaning appear to be able to generate a relatively strong jaw-closing force. The generation of the jaw-closing force in neonates may contribute to the proper development of the oro-motor system.

During postnatal development, the percentage of type I JCMNs increased by up to $82 \%$ at 16-27 days. This increase has been shown in adult hypoglossal motoneurons that mainly exhibit a decrementing firing pattern similar to type $\mathrm{I}^{16)}$. The type I firing property leads to an initial high firing rate of JCMNs in response to excitatory inputs, even when the inputs are not strong. Furthermore, the incidence of an extra spike that also produces an initial high firing rate increased in the JCMNs with age. The initial high firing rate of JCMNs would enable the fast rise of the jaw-closing force necessary to chew food because insertion of a single extra action potential in a low frequency spike train of a spinal motoneuron can cause the marked, long-lasting tension enhancement produced by the muscle fibers innervated by the motoneuron ${ }^{22)}$. These developmental changes in firing properties of JCMNs, as well as the high maximal firing rate observed throughout the postnatal development, may contribute to the transition from suckling to chewing.

\section{Acknowledgments}

This work was supported by the Project to Establish Strategic Research Center for Innovative Dentistry and grantsin-aid for scientific research (Nos. 20592186 and 23592750) by MEXT.

\section{References}

1 ) Ahlgren J. Mechanisms of mastication. Acta Physiol Scand, 1966. 24: Suppl. 44, 1-109.

2 ) Hidaka O, T Morimoto, Y Masuda, et al. Regulation of masticatory force during cortically induced rhythmic jaw movements in the anesthetized rabbit. J Neurophysiol, 1997. 77: 3168-79.

3 ) Inoue T, T Kato, Y Masuda, et al. Modifications of masticatory behavior after trigeminal deafferentation in the rabbit. Exp Brain Res, 1989. 74: 579-91. 
4) Plesh O, B Bishop, and W McCall. Effect of gum hardness on chewing pattern. Exp Neurol, 1986. 92: 502-12.

5 ) Yamaoka A, T Inoue, S Hironaka, et al. Postnatal changes in electrophysiological properties of rat jaw-closing motoneurons. Showa Univ J Med Sci, 2005. 17: 71-79.

6 ) Nakamura S, T Inoue, K Nakajima, et al. Synaptic transmission from the supratrigeminal region to jawclosing and jaw-opening motoneurons in developing rats. J Neurophysiol, 2008. 100: 1885-96.

7 ) Gemba-Nishimura A, T Inoue, S Nakamura, et al. Properties of synaptic transmission from the reticular formation dorsal to the facial nucleus to trigeminal motoneurons during early postnatal development in rats. Neuroscience, 2010. 166: 1008-22.

8 ) Aghajanian GK and K Rasmussen. Intracellular studies in the facial nucleus illustrating a simple new method for obtaining viable motoneurons in adult rat brain slices. Synapse, 1989. 3: 331-8.

9 ) Mizuno N, A Konishi, and M Sato. Localization of masticatory motoneurons in the cat and rat by means of retrograde axonal transport of horseradish peroxidase. J Comp Neurol, 1975. 164: 105-15.

10) Limwongse V and M DeSantis. Cell body locations and axonal pathways of neurons innervating muscles of mastication in the rat. Am J Anat, 1977. 149: 477-88.

11) Sessle BJ. Identification of alpha and gamma trigeminal motoneurons and effects of stimulation of amygdala, cerebellum, and cerebral cortex. Exp Neurol, 1977. 54: 303-22.

12) Inoue T, S Itoh, M Kobayashi, et al. Serotonergic modulation of the hyperpolarizing spike afterpotential in rat jaw-closing motoneurons by PKA and PKC. J Neurophysiol, 1999. 82: 626-37.

13) Nishimura Y, PC Schwindt, and WE Crill. Electrical properties of facial motoneurons in brainstem slices from guinea pig. Brain Res, 1989. 502: 127-42.

14) Gueritaud JP. Electrical activity of rat ocular motoneurons recorded in vitro. Neuroscience, 1988. 24: 837-52.

15) Nunez-Abades PA, JM Spielmann, G Barrionuevo, et al. In vitro electrophysiology of developing genioglossal motoneurons in the rat. J Neurophysiol, 1993. 70: 1401-11.

16) Viana F, DA Bayliss, and AJ Berger. Repetitive firing properties of developing rat brainstem motoneurones. J Physiol, 1995. 486 ( Pt 3): 745-61.

17) Chandler $\mathrm{SH}, \mathrm{CF}$ Hsaio, T Inoue, et al. Electrophysiological properties of guinea pig trigeminal motoneurons recorded in vitro. J Neurophysiol, 1994. 71: 129-45.

18) Fulton BP and K Walton. Electrophysiological properties of neonatal rat motoneurones studied in vitro. J Physiol, 1986. 370: 651-78.

19) Hille B. Ionic Channels of Excitable membranes. Vol. 3rd ed. 2001, Sunderland, MA: Sinauer.

20) Gallender D. Eating handicaps, illustrated techniques for feeding disorders. 1979, Springfield: Charles C Thomas.

21) Ainamo J. Prenatal occlusal wear in guinea pig molars. Scand J Dent Res, 1971. 79: 69-71.

22) Burke RE, P Rudomin, and FE Zajac, 3rd. Catch property in single mammalian motor units. Science, 1970. 168: 122-4. 